$\$$ sciendo

\title{
Naïve Realism and the Problem of Causation
}

\author{
Michael Sollberger \\ University of Lausanne \\ Disputatio Vol. 3, No. 25 \\ November 2008
}

DOI: $10.2478 /$ disp-2008-0007

ISSN: 0873-626X 


\title{
Naïve realism and the problem of causation
}

\author{
Michael Sollberger \\ University of Lausanne
}

\begin{abstract}
In the present paper, I shall argue that disjunctively construed naïve realism about the nature of perceptual experiences succumbs to the empirically inspired causal argument. The causal argument highlights as a first step that local action necessitates the presence of a type-identical common kind of mental state shared by all perceptual experiences. In a second step, it sets out that the property of being a veridical perception cannot be a mental property. It results that the mental nature of perceptions must be exhausted by the occurrence of inner sensory experiences that narrowly supervene on the perceiver. That is, empirical objects fail directly to determine the perceptual consciousness of the perceiver. The upshot is that not only naïve realism, but also certain further forms of direct realism have to be abandoned.
\end{abstract}

\section{Keywords}

Naïve realism, causation, perceptual consciousness, disjunctivism, hallucination.

\section{Introduction}

Disjunctivism is currently one of the most hotly debated topics in the philosophy of perception. This is so because defenders of disjunctivism, such as for example Hinton 1973, Fish 2008, Langsam 1997, Martin (1997; 2004; 2006) and Snowdon 1981, put forward an intriguing theory of naive realism that is supposed to secure direct perceptual contact with the mind-independent empirical world, i.e. there are at least some experiential events by means of which everyday physical objects are presented to the perceiver in a metaphysically transparent way. ${ }^{1}$ Disjunctivism is said to have the further attractive

\footnotetext{
${ }^{1}$ As made clear by Byrne \& Logue 2008, disjunctivism is not a single theory but comes in various forms. In this paper, I shall be concerned only with what Byrne \&

Disputatio, Vol. III, No. 25, November 2008
} 
virtue that it is the only theory which can do justice to the phenomenology of perception, namely as the transparent awareness of presently existing empirical objects and their properties (see Martin's 2004b theses of 'Transparency' and 'Actualism'). Moreover, disjunctivism is thought to circumvent the threat of a 'veil of perception' with its ensuing epistemological worries. So, it is no surprise that a huge amount of recent work has been dedicated to discussing the disjunctive theory of perceptual content.

In the present paper, I shall argue that enthusiasm for disjunctivism is misplaced. In particular, disjunctivism is doomed as soon as we focus on the causal argument. Properly understood, the causal argument makes plain three things: i) local action necessitates the presence of a common type-identical mental state shared by all perceptual experiences, be they veridical or delusory; ii) this type-identical mental state constitutes the most fundamental kind of perceptual experiences; iii) it further yields the elimination of the property 'being a veridical perception' qua mental property.

As a possible first response, disjunctivists may try to bypass such a common kind throughout. However, I shall highlight that such an attempt inevitably amounts to spooky action at a distance. An alternative disjunctivist rejoinder consists in saying that such a common kind in perception and hallucination is still compatible with disjunctively construed naïve realism and does not yet entail the idea of the 'highest common factor' (see Martin 2004). I shall show that this second suggestion ultimately fails; for the common mental property screens off the special property 'being a perception', such that the special property cannot be genuinely mental. Accordingly, I finally argue that we have to abandon disjunctively construed naive realism altogether in favour of a genuine common factor view of perceptual consciousness.

\section{Disjunctivism and naïve realism}

Basically, disjunctivism originates with the idea that look-statements, such as ' $x$ looks some way to $S$ ', are sentences with a disjunctive form. Their correct analysis is 'Either $S$ perceives $x$ or it merely seems to $S$ as though there were an x' (see Hinton 1967: 12 and Snowdon 1981:

Logue call 'metaphysical disjunctivism', i.e. disjunctivism about the nature of perceptual experiences. Accordingly, I shall not pay attention to 'epistemic disjunctivism' as advocated by McDowell 1998. 
185). The main reason for endorsing disjunctivism is to defend naïve realism (NR) against the argument from illusion and from hallucination (cf. Martin (1997; 2004; 2006) and Smith 2002). According to naïve realism, the perceiving subject $S$ stands in a metaphysically transparent awareness-relation to mind-independent objects - the concrete individuals and their properties. These objects of perception are the literal constituents of the perceptual episode. Correspondingly, empirical objects are said to determine and constitute the phenomenal character of $S$ 's experience in perception (see Campbell 2002: 116 and Martin 1997: 83-84). That is, by perceiving the mindindependent object $x, S$ is phenomenally aware of $x$ and its properties. Perceptions are essentially relational states of affairs and thus necessarily world-including. Given that hallucinations are not objectdependent, they are conceived of as mental states of a distinct most fundamental kind. ${ }^{2}$ The notion of 'most fundamental kind' is meant to capture the real essence or identity of the mental state. Thus, NR claims that perceptions and hallucinations have different metaphysical natures: whilst perceiving an empirical object $x$, the subject $S$ is in a mental state of fundamental kind $K$, and no experience in the absence of $x$ could be of kind K (see Martin 2004: 39).

Thus conceived, disjunctivism is incompatible with what Martin (2004: 40) calls the 'Common Kind Assumption' (CKA). According to CKA, whatever fundamental kind $\mathrm{K}$ of mental state occurs when $S$ is perceiving $x, \mathrm{~K}$ can occur whether $S$ is perceiving or hallucinating $x$. One impetus for CKA is the Cartesian view according to which we should individuate kinds of mental states according to subjective and introspective evidence. More precisely, disjunctivists refuse to admit that if two experience-tokens are, after close and attentive introspection, subjectively indiscriminable from each other, then they have to belong to the same most fundamental mental kind $\mathrm{K} .{ }^{3}$ Over and above these considerations, disjunctivists like Martin (2004: 39; 2006: 357) subscribe to 'Experiential Naturalism' (EN): they grant that perceptual experiences are events embedded in the natural causal

\footnotetext{
2 There is no consensus among disjunctivists whether illusions belong to the good or bad cases. For reasons of simplicity, I will not address this topic here and only work with hallucinations.

${ }^{3}$ This formulation is rough-and-ready and would require further refinement. Although, for present purposes, it provides a sufficiently clear idea of the Cartesian principle of type-individuation of mental kinds.
} 
network. That is, perceptual experiences are subject to physical and psychological causes - they are not nomological danglers.

To summarize, the flavour of disjunctivism we are concerned with in this paper endorses both NR and EN. Given that disjunctivists think that NR entails the denial of CKA, i.e. NR $\rightarrow \sim \mathrm{CKA}$, they also endorse $\sim$ CKA. ${ }^{4}$ In what follows, I shall show that on the most plausible reading of the causal argument, it ultimately follows that the causal argument entails CKA, and hence, by modus tollens, $\sim \mathrm{NR}$.

\section{The causal argument}

The causal argument (CA) is as follows: Data from neurobiology strongly suggest that a neurosurgeon can possibly evoke multi-modal hallucinations in a subject $S$ which are subjectively indistinguishable from corresponding veridical perceptions (see Bickle \& Ellis 2005). ${ }^{5}$ The neurosurgeon may achieve this through suitably performed artificial micro-stimulation of $S$ 's brain. If so, appropriate causal stimulation of $S$ 's brain turns out to be a minimal sufficient condition for a particular hallucination (h) to occur. Let us suppose that $S$ hallucinates a red apple in front of her and this adequately characterizes the what-it-is-likeness of $S$ 's hallucination. Importantly, this kind of awareness involved in hallucination narrowly supervenes on $S$ 's total brain state, for it fails to depend on currently present worldly objects. That is to say that the occurrence of $h$ requires no more than $S$ 's brain being in a certain state. In brief, $h$ is an intra-mind affair.

In a next step, notice that $S$ can be in the same total brain state whilst veridically perceiving a red apple. Albeit having a distinct aetiology, the causal chains leading to the perception $(p)$ of the apple and to the subjectively indistinguishable hallucination as of an apple partly overlap, for they both contain $S$ 's total brain state as a type-identical proximate cause. It results that the causal stimulation of $S$ 's brain must

\footnotetext{
${ }^{4}$ I will not enter the debate whether to adopt NR together with the denial of CKA is to count as a genuine form of disjunctivism (cf. Byrne \& Logue 2008). In any case, it reflects Martin's 2004 view, and Martin provides the most elaborated answer to the causal argument from a disjunctivist perspective (see also Langsam 1997 for a discussion of the causal argument). That is why my discussion will focus mostly on Martin's disjunctivism.

${ }^{5}$ That is, the artificially evoked hallucination as of an $x$ is, based on introspective means alone, not knowably distinct from the corresponding veridical perception of an $x$.
} 
also be minimally sufficient for bringing about $h$ in perception. Therefore, $h$ must be co-present with $p$ whilst $S$ perceives the red apple. But then, plausibly, given that $h$ ontologically grounds $S$ 's seeming awareness of the apple, $h$ has also to be sufficient for ontologically grounding the phenomenology of $p \cdot{ }^{6}$ As a consequence, the hallucinatory experience-token $h$ pre-empts $p$ from explaining ontological, causal and psychological facts concerning $S$ 's mental state of perceiving the red apple and thereby renders $p$ explanatorily idle with respect to this token of experience. Finally, $p$ 's explanatory idleness renders $p$ useless. Hence, in contradiction to naïve realism, $p$ has to be eliminated at the tokenlevel of perceptual experience, for it highlights that it cannot be the empirical object $x$ - the red apple in our case - that determines the phenomenal character of $S$ 's perception.

This upshot yields two insights: First, CKA is true, for $h$ constitutes the most fundamental mental kind common to both $p$ and $h$. Hence, by modus tollens, the brand of perceptual token-externalism embraced by NR must be false, since, according to disjunctivists, naïve realism implies the denial of CKA. And second, the causal argument leads to the elimination of the property 'being $p$ ' qua mental property. If correct, this result is relevant for each theory of perception that tries to combine the idea of a type-identical mental state common to both perceptions and hallucinations with the idea that empirical objects and their properties can nonetheless directly determine $S$ 's perceptual consciousness in veridical perception. Typically, direct realists like Dretske 1995 and Johnston 2004 defend such a theory. That is, if the causal argument is sound, such forms of direct

\footnotetext{
${ }^{6}$ Note that the causal argument does not presuppose that $h$ has exactly the same phenomenal character as $p$. Crucially, most proponents of naïve realism put forward phenomenal irrealism about hallucinations. That is, they deny that hallucinations have phenomenal character at all, whilst agreeing that they certainly seem to (Fish 2008: 159). For instance, by referring to higher-order beliefs, Fish 2008 tries to explain how $S$ can mistake a mental state, which is intrinsically distinct from a perception, for such a perception. Naïve realists thus accept that there is something it is like for $S$ to undergo a hallucination, i.e. that hallucinations can have the same phenomenology as perceptions, but reject the idea that $S$ 's inability to distinguish by introspective means $h$ from $p$ is due to the fact that hallucinations and perceptions share the same basic phenomenal character. Given that all the causal argument needs to say is that $h$ and $p$ have the same phenomenology for $S$, the causal argument does not beg the question against naive realists who are driven by phenomenal irrealism about hallucinations.
} 
realism are, alongside with naïve realism, also jeopardized. I shall come back to this issue further on.

Moreover, the causal argument does not take a stand on how to construe the metaphysical nature of the fundamental kind mentioned in CKA. For all we know so far is that the fundamental common kind $h$ might be metaphysically realized by the state of affairs of $S$ 's being related to sense-data, qualia, intentional objects, ways of sensing, higher-order attitudes, or what have you - they may all equally fill this position. CKA only requires $h$ to be a narrowly supervening inner state of $S$ whose identity is not constitutively dependent on x's actual presence. In the remainder of the text, I shall i) comment in more detail on the controversial premisses of the causal argument, ii) discuss Foster's 2000 and Martin's 2004 rejoinders to it and iii) stress that the causal argument also rules out certain further accounts of direct realism over and above naïve realism.

\section{The causal argument defended}

It is important to note that the causal argument decisively relies on a causal principle $(\mathrm{CP})$ that needs explication. Here it is:

(CP) If there occurs a causal chain $\Phi$ that is made up of the causal links $\left[\mathrm{C}_{\mathrm{c}-1}, \mathrm{c}\right]$ and whose proximate cause $\mathrm{c}$ is minimally sufficient for causing an effect $\Omega$, and if there is an extended, partly overlapping causal chain $\Psi$ that is made up of the causal links $\left[\mathrm{c}_{\mathrm{c}-\mathrm{x}}, \ldots, \mathrm{c}_{\mathrm{c}-1}, \mathrm{c}\right]$, then it is nomologically impossible that $\Psi$ occurs without causing $\Omega$.

CP's rationale is backed up by current research in special relativity. It is common ground in philosophy of science that action at a distance is excluded by special relativity and its well known principle of local action (see Einstein 1948: 321-322; Howard 1985: 187-188). Correspondingly, a theory of causation should stick only to local causation and exclude the possibility of unmediated action at a distance. Over and above that, it is important to realize that the causal principle also fits with the working hypothesis currently applied by neurobiologists; they pay attention to local causation and internal states and do not pursue the possibility of action at a distance in their explanations. That is, it is fundamental to the currently well established method of empirical research on perception that perceptual states causally depend on proximate stimulations and internal input, such that the 
effects of distal causes are completely accounted for by their proximate causes (this point has been forcefully argued for by Burge 2005).

Furthermore, it is important to note that the causal principle does not entail that $\Omega$ is everything $\Psi$ can bring about. It is logically possible for the extended causal chain $\Psi$ to bring about an extra effect $\Omega *$ together with $\Omega$. This admits that remote causes such as $\mathrm{C}_{\mathrm{c}-\mathrm{x}}$ can play a role in leading to a supplementary effect $\Omega *$ whose identity fails to be fully accounted for by the proximate cause c. So put, the causal principle allows that $p$ and $h$ might coexist when $S$ veridically perceives $x$. However, in order not to violate the principle of local action, the additional effect $\Omega *$ must be epiphenomenal. Glossed in concrete terms, CP entails that $S$ 's total brain state cannot bestow extra causal powers on $p$ over and above the causal powers conferred on the common effect $h$. This yields severe troubles for disjunctivists insofar as they have to individuate perceptual states by means of relational properties. I shall elaborate on this issue below.

However, local causation has been taken to imply something stronger than CP. According to Robinson $(1985 ; 2001)$, if c is minimally sufficient for $\Omega$ in $\Phi$, then $\Psi$ must cause $\Omega$ too, and $\Omega$ is everything $\Psi$ can bring about. That is, the type-identical experience $h$ is everything S's type-identical brain state can give rise to. This excludes the possibility that, in veridical perception, $p$ might occur together with $h$ as a supplementary effect.

To my mind, Robinson's suggestion is wanting for two reasons. First, we have a cross-connection of the neurobiological cause, namely $S$ 's brain state, with $S$ 's perceptual experience. Unlike physical-to-physical causation, though, cause and effect cannot be observed independently from each other in perception. ${ }^{7}$ Whilst perceiving $x, S$ perceives the physical cause but undergoes its mental effect. Naïve realists may thus simply object that Robinson's suggestion in the psychophysical context is impermissible. Second, it begs the question against disjunctivism for the reason that its plausibility rests on internalist intuitions. The externalist framework of naïve realism has it that the perceptual mind is not in the head (cf. McDowell 1992: 36). Perception necessitates the interaction of a broad intricate network that comprises both $S$ and $S$ 's environment. If so, then it seems natu-

\footnotetext{
${ }^{7}$ Hume 1740 famously argued for this independence between cause and effect. See also Hinton's (1973: 75-93) disjunctivist defence of naïve realism against the causal principle and Robinson's 1985 reply.
} 
ral to suppose that the artificial stimulation of $S$ 's brain state does not have to give rise to exactly the same mental effect $h$ as in genuine perception; after all, brain-activation constitutes just one single piece within this broad, complex network. Hence, Robinson's suggestion is plausible only if one makes the internalist assumption that perception exclusively happens inside $S$ 's skin. This is obviously begging the question and an attack on naïve realism should, therefore, sidestep Robinson's suggestion.

One might object that, like Robinson's suggestion, the way I make use of $\mathrm{CP}$ in the context of perception also hinges on internalist assumptions and, therefore, also begs the question against naïve realism since it assumes that $h$ exclusively happen inside $S$ 's skin. This arrow does not, however, hit the bull's-eye. Whereas Robinson's suggestion is question-begging because it rests on internalism about perceptual experiences in general, the use of $\mathrm{CP}$ amounts to internalism about hallucinatory experiences only, and not to internalism about perceptions. And surely, this internalism about hallucinations is harmless, for naïve realists readily accept that $h$ is an inner state that narrowly supervenes on $S .{ }^{8}$ Henceforth, I shall therefore take for granted that $\mathrm{CP}$, as formulated above, is the right principle for the causal argument to work with.

With these clarifications at hand, let us discuss Foster's (2000: 2343) objection to the causal argument. ${ }^{9}$ As a start, note that Foster accepts the principle 'same proximate cause, same immediate effect'; to wit, he accepts CP. Given this acceptance of CP together with the fact that $p$ must constitutively depend on $x$ if naïve realism is to be true, the following worry comes up:

So by what mechanism does the mind adjust its response to fit the character of the remote cause [x]? How, as it were, does the mind know whether the central-nervous process was caused in the normal or the artificial way before selecting its response? (Foster 2000: 28).

\footnotetext{
${ }^{8}$ For instance, Martin (2004: 58) writes that ' $[. .$.$] hallucinations are "inner ex-$ periences". We have the conception that the occurrence of such events imposes no additional condition on the world beyond the subject's putative state of awareness.'

${ }^{9}$ Just note that even if Foster 2000 alleges that the causal argument against naïve realism is flawed, he himself does not endorse naïve realism.
} 
As Foster (2000: 37) rightly acknowledges, it is empirically implausible to suggest that $S$ 's brain state could preserve information about the causal aetiology that has induced its actual realization; such a thought is thoroughly undermined by current empirical findings in the neural sciences (see Gazzaniga, Ivry \& Mangun 2002). So, referring to brain states that could 'know' their distal causal antecedents is no serious option for naïve realists to side-step the causal argument.

Instead, Foster advances the following claim: the empirical object $x$, in addition to initiating the whole causal chain with all the intermediary causal steps, can directly causally contribute to $S$ 's brain state and thus directly determine what kind of mental state $S$ is in (see also Hinton 1973: 75). $X$ is considered both as a remote and as a proximate cause of $p$, since $x$ and the whole causal aetiology it initiates directly causally enter into $S$ 's brain state.

When the psychological outcome is about to occur, the factors which directly contribute, causally, to its occurrence and character include not just the current state of the brain, but also certain aspects of the preceding causal process, including, crucially, certain aspects of the way in which the neural process leading up to the realization of the brain state has itself been brought about. (Foster 2000: 36)

Thus conceived, the proximate cause of $p$ and $h$, viz. $S$ 's brain state, turns out to be type-distinct in perception and hallucination, since the whole causal aetiology, which differs in $p$ and $h$, acts as the proximate cause. From this follows that, according to Foster, the causal argument is unsound because $S$ 's brain state is not a type-identical proximate cause common to perception and hallucination. If true, then Foster's suggestion is in keeping with $\mathrm{CP}$, because type-distinct proximate causes lead to type-distinct immediate mental effects in perception and in hallucination. This is how Foster is able to deny, on behalf of naïve realism, that $h$ must be co-present with $p$ in perception.

It is important to realize that Foster's main concern is with causation and, in particular, with how $x$ can have any direct causal bearing on $p$. As he makes clear, it would be intellectually unsatisfying if naïve realists simply answered the causal argument by pointing out that there was nothing logically incoherent in insisting that the same type of neural brain state could give rise to different types of mental states. This would not explain at all why there should be such a difference in mental outcome and, consequently, leave the position of naïve realists 
incredible (Foster 2000: 35). This means that mere logical possibility of external type-individuation of perceptions and hallucinations is really not the point at issue. Rather, emphasis is placed on how causal interaction between $x$ and $S$ has to be conceived of in order to do justice to the fact that the occurrence of a perception constitutively depends on the presence of $x$, whereas a hallucination fails to do so. ${ }^{10}$

However, as Foster (2000: 37) himself concedes, such a causal mechanism seems bizarre. In fact, in addition to being bizarre, I think the idea of such a causal mechanism is far-fetched and should be rejected even by naïve realists, for i) it lets in unmediated action at a distance and ii) it is highly implausible from an empirical point of view. As regards i), Foster writes that ' $[. .$.$] earlier events may have a$ direct causal influence on what happens after a temporal interval' (Foster 2000: 37). This means that even if the distal cause $x$ is temporally earlier than $S$ 's brain state that is supposed to engender $p$, this does not hinder $x$ from directly causally influencing $S$ 's spatially remote brain state. Clearly, this is tantamount to let in action at a distance. Yet, as settled above, action at a distance has to be eschewed because it violates the principle of local action.

Of course, it is not a priori that action at a distance has to be excluded in the psycho-physical domain of perception. The reason why naïve realists are nonetheless well advised to avoid this position brings us to point ii): Neuroscientists do not appeal to action at a distance in order to explain how, for example, the neural firing patterns of $S$ 's brain state give rise to $S$ 's perceptual experiences. They only refer to local, neurological causes, and this methodology is amply justified by current research practice (see Burge 2005 for this point). As a consequence, naïve realists would be in a situation that is dialectically very weak; for they would have to argue for the falseness of currently well established empirical data in the neurological realm. This would seem ad hoc and unjustified, since naïve realists would insist, on purely conceptual grounds, that action at a distance for neurological causes occurs in spite of all the empirical evidence to the contrary. That is to say that the commitment to action at a distance in the perceptual

\footnotetext{
${ }^{10}$ Note: No one in the debate disputes that $h$ and $p$ are type-distinct states, that much is uncontroversial. What really is controversial is whether perceptions and hallucinations are type-distinct as regards their mental nature.
} 
realm is clearly a non-starter. Hence, disjunctivists cannot take refuge in Foster's proposal and the causal argument is still safe. ${ }^{11}$

We have seen above that naïve realists, such as Martin, subscribe to 'Experiential Naturalism', i.e. they are willing to embed perceptual experiences in the causal natural network. Indeed, the actual understanding of the causal natural network, within which perceptual experiences take place, is essentially tied to the background of special relativity and the neurosciences. As seen, this empirical background eschews action at a distance. It is therefore part of the spirit of EN that naïve realism ought to be spelled out without introducing such bizarre causal mechanisms. In brief, action at a distance does not square with EN. Naïve realists thus have to accept the co-presence of $p$ and $h$ in perception and show that this co-presence is not yet sufficient for establishing CKA.

Martin's 2004 objection against the causal argument takes exactly this line, that is, he thinks the co-presence of $p$ and $h$ in perception is compatible with naïve realism. Basically, his idea is this: Although perception and hallucination share a common mental kind $h, h$ does not constitute the most fundamental kind in perception. $S$ 's hallucinatory experience as of an $x$ is fundamentally of kind $h$, and $S$ 's veridical experience of $x$ is also of kind $h$, but not fundamentally, because it is fundamentally of kind $p$. So far, so good. What, then, is Martin's strategy for resisting the upshot of the causal argument?

Martin's overall line of attack is quite complex and involves considerations about the concept of sensory experience which I cannot deal with here in detail due to lack of space (see Byrne \& Logue 2008 and Siegel $(2004 ; 2008)$ for a critical discussion of Martin's view). In a nutshell, Martin (2006: 369) claims that there is nothing more to the phenomenal character of $h$ than that of being subjectively indiscriminable from the corresponding veridical perception $p$. The only positive mental property of $h$ is epistemically and not phenomenally or metaphysically characterized: $h$ is not knowably different from $p$ as

${ }^{11}$ A further drawback of Foster's theory stems from the way he conceives of the causal mechanism that leads to hallucinations: any deviance in the 'normal' causal chain that leads to perceptions has a merely negative influence on the psychological outcome, to the effect that $h$ takes place in lieu of $p$. As Martin (2004: footnote 16) points out, such a view is highly problematic, for it allows 'the absence of a specific causal factor itself to count as a distinctive causal factor'. 
far as $S$ can tell through introspection alone. ${ }^{12}$ Accordingly, $h$ is said to belong to the fundamental kind 'being indiscriminable from $p$ ' and $p$ to the fundamental kind 'being a veridical perception of $x$ '. Given that it is trivial that $p$ is not discriminable from itself, $h$ is common to both experiences without constituting their most fundamental common mental kind. ${ }^{13}$

Let us assume that Martin's conception is in line with naive realism and also with the denial of CKA. Still, there is the problem of explanatory pre-emption: the common epistemic property of 'being indiscriminable from $p$ ' screens off the special property of 'being $p$ '. That is, the common property screens off the special one. The key thought behind this can be stated by what I call the 'Principle of Screening Off' (PSO):

(PSO) If there are two tokens of perceptual experiences $e_{1}$ and $e_{2}$ which are subjectively indiscriminable for $S$ and which have a kind of common mental property $P$ that is in itself sufficient for explaining the mental nature of $e_{1}$, and $e_{2}$ is supposed to have a special mental property $Q$ which $e_{1}$ lacks, then $P$ screens off $Q$ from playing any distinctive explanatory role for the mental nature of $e_{2}$. Hence, $Q$ becomes explanatorily idle with regard to the mental nature of $e_{2}$.

It is hard to see how PSO could be resisted. Again, note that the causal argument in general and PSO in particular do not assert that the property of being a veridical perception per se is explanatorily redundant. It may be that, in order to explain some of $S$ 's actions, explanation is conceptually tied to distal success and not only to proximate behaviour. That is, 'being $p$ ' may be still useful for explaining why, say, $S$ reaches for the apple in front of her upon having visually perceived it. In such cases, the explanans may be necessarily relational, so that the property 'being $p$ ' may enjoy an explanatory

\footnotetext{
${ }^{12}$ A precision: Martin's 2004 purely epistemic characterization of $h$ applies only to cases of 'causally matching hallucinations', i.e. a hallucination as of an $x$ that has been brought about by exactly the same proximate causes as the corresponding perception of an $x$. As to the nature of all the other, causally non-matching hallucinations, Martin remains agnostic.

${ }^{13}$ A strategy in a similar spirit to Martin's can also be found in Pitcher (1971: 56-57) where he argues that naive realism is compatible with $\mathrm{CP}$, for although $\mathrm{CP}$ 'may be true for every cause-and-effect pair, [it] it is not true for them under every description.'
} 
potential that $h$ lacks (see Peacocke 1993). Nonetheless, this only applies to relational facts between $S$ and $S$ 's environment and not, as it is required by naive realism, to the relational nature of perceptual consciousness itself. This means that such externalism about explanation, which relates to the phenomena subsequent on perception, fails to constitute a genuine counterexample to PSO.

Moreover, in order not to beg the question against the naïve realists' treatment of hallucinations, PSO does not imply that the common mental property $P$ be genuinely phenomenal. $P$ 's correct characterization might for instance be epistemic in the following sense: $S$ has the false higher-order belief that she is self-aware of an experiential, first-order perceptual experience of an $x$, and it is this erroneous higher-order epistemic perspective on her own mind that is, in itself, sufficient for grounding S's phenomenology of $e_{1}$ (see Fish 2008 and Martin 2006 for more on this topic). In opposition to the mental nature of $Q$ that may be genuinely phenomenal, the mental nature of $P$ might be purely epistemic. It is perfectly compatible with the above formulation of PSO that this fundamental ontological difference between $e_{1}$ and $e_{2}$ does not necessarily have to show up in $S$ 's phenomenology, such that $e_{1}$ and $e_{2}$ can be subjectively indiscriminable for $S$.

So let us turn to Martin's objection against the validity of the 'Principle of Screening Off'. Here is the relevant passage:

[W] hat seems key is the thought that the explanatory properties of the common property are derivable a priori from the special property. We can tell that the common property must be correlated with the outcome just from knowing what the special property can otherwise explain. Hence, there is a reason to think that the property of being indiscriminable from an $\mathrm{F}$ has an explanatory potential which is dependent on the explanatory potential of being an F. [...] So we may conclude that ... cases of inherited or dependent explanatory potential offer us exceptions to the general model of common properties screening off special ones. (Martin 2004: 70) ${ }^{14}$

Hence, according to Martin, the scope of PSO does not encompass our case because the explanatory potential of $h$ can be derived a priori from $p$. If one wants to block Martin's attack against PSO, there are

${ }^{14}$ My PSO does not equal Martin's 2004 more general account of 'screening off', for PSO relates only to tokens of experiences and their mental nature. Yet, this difference is not crucial for the present argument. 
two ways one can pursue. First, given that Martin's objection to PSO decisively hinges on his epistemic account of hallucination, one may directly dispute this conception. In fact, this has already been forcefully done by, among others, Hawthorne \& Kovakovich 2006 and Siegel $(2004 ; 2008)$. I will not, at present, get into the points stressed by these criticisms. Instead, for the sake of argument, let us suppose that Martin could surmount these objections. If so, the second option for countering Martin's objection to PSO is to offer better reasons than Martin offers as to why there is such a correlation between the explanatory properties of $p$ and $h$. In what follows, I shall argue that there are in fact such superior reasons.

Roughly, the key idea is this: at the token-level, the property of being $P$ can be demonstrated to be non-phenomenological, nondoxastic, non-epistemic and non-causal. Firstly, 'being $p$ ' is nonphenomenological because $p$ and $h$ are per definitionem subjectively indistinguishable from each other. There is no noticeable difference of what it is like for $S$ to be in $p$ or $h$. Given that $p$ and $h$ must occur together in perception and given that $h$ undertakes the whole job of grounding and explaining $S$ 's phenomenology of hallucinations, $P$ must thus be uncoupled from phenomenology.

Secondly, by applying Dunn's (2008: 388-392) arguments to our present issue, one can persuasively show that as soon as one accepts that $h$ must occur in perception alongside with $p, h$ does the whole work for $S$ 's doxastic and epistemic awareness. ${ }^{15}$ That is, all of $S$ 's perceptual beliefs present in veridical perception can be accounted for by $h$, such that $p$ cannot play any doxastic role for the perceptual beliefs caused by perceptual awareness. Equally, Dunn persuasively makes the point that $h$ does all the justificatory work needed in veridical perception. Hence, in addition to being phenomenologically inert, $p$ fails to play any doxastic or epistemic role for $S$ 's cognition.

And finally, $p$ is epiphenomenal. The causal principle implies that $p$ fails to have additional causal powers which are not already had by $h$. That is, the inner experience $h-$ an intrinsically non-relational perceptual seeming — does the whole causal job. Given that naïve realism individuates $p$ relationally with reference to $x$, it follows that

${ }^{15}$ To be sure, Dunn's 2008 discussion is directed at Johnston's 2004 direct realism and not at naïve realism. However, given that Martin accepts that $h$ occurs together with $p$ in perception, nothing hinders that Dunn's arguments can be applied, mutatis mutandis, to our present discussion. 
the relational property of being a veridical perception is causally impotent. Hence, there is no causal work left for naïve perceptual content.

At present, it is important to realize that even if, let us assume for the sake of argument, naïve realists could nevertheless come up with a theory that equipped the relational property of being $p$ with a role to play in $S$ 's perceptual awareness, the very fact that $p$ must be epiphenomenal proves, on its own, highly problematic for naïve realists as I shall explain. First of all, note that naive realists are in the same predicament as externalists when they have to account for the causal efficacy of perceptual states qua contentful states. If the relationally individuated content-property of being $p$ is causally impotent, only intrinsic properties of $S$ can do the causal work. This means that, say, $S$ 's action of picking up the apple, forming the belief that the apple is in front of her etc. cannot be causally explained by $S$ 's being phenomenally appeared to the apple, but only by $S$ 's being in the internal brain state. This is awkward, since the understanding we have of ourselves as rational agents crucially hinges on the fact that we act in agreement with intentional properties of our mental states (see for example Fodor (1987: chapter 2) for this point). Naive realism is obviously at odds with perceptual epiphenomenalism. And as a second point, one may insist that entering into causal chains seems to be the sole way for physical entities to become epistemically salient to us cognizers (Shoemaker 1980). Yet, the epiphenomenal property of being $p$ would be unable to do this. So, this already warrants its elimination. In sum, then, this argument highlights that even if naive realists could somehow show the relational property of being $p$ to be doxastically or epistemically active for $S$ 's perceptual awareness, the serious problem of perceptual epiphenomenalism would remain untouched.

Against this background, it becomes completely unclear as to what the alleged property of being $p$ should be. At any rate, it does not look like a form of awareness at all, for it plays no role in $S$ 's cognitive architecture. That is, we are left with no rationale for maintaining $p$ since $h$ can undertake the whole job of adequately explaining phenomenological, doxastic, epistemic and causal facts of perceptual experience-tokens. Therefore, the best reason we have for making sense of the explanatory correlation between $p$ and $h$ is that they are mentally alike, and not, as Martin wants us to swallow, that the explanatory potential of $h$ depends on the property of being $p$. That is to say that as regards to mentality, there is no real ontological distinction 
between $p$ and $h$, for the property 'being a perception' is eliminated qua mental property. ${ }^{16}$ This settles that $p$ and $h$ belong, pace Martin, to the same most fundamental mental kind. What is more, it is no surprise that such a derivability-relation holds if CKA obtains. Martin's worry is perfectly compatible with the view that 'being $p$ ' is not a mental property, but a complex state of affairs that is composed out of the inner experience $h$ and the extraneous, causally related nonmental fact of $x$ 's obtaining. ${ }^{17}$ Cases of inherited explanatory potential constitute thus no exception to PSO, i.e. we have no reason to hold on to the property 'being $p$ ' qua mental property. Therefore, the causal argument is still safe. This is bad news for naive realists, for it means that CKA, and hence $\sim \mathrm{NR}$.

\section{Conclusion}

To conclude, let me briefly stress one last point. As formulated above, the causal argument also demonstrates that the empirical object $x$ that exists hic et nunc is unable to have a direct impact on $S$ 's perceptual consciousness in veridical perception. This is so because the presence of $h$ in perception inevitably leads to $p$ 's elimination. This upshot not only goes against naïve realism, but also against most forms of direct realism, such as they have been advocated by Dretske 1995 and Johnston 2004, among many others. Basically, these direct realists seek to combine the existence of a type-identical common mental factor in perception and hallucination with the idea that 'seeing goes all the way out to the things seen, the things with which it acquaints the subject' (Johnston 2004: 139). ${ }^{18}$ But by eliminating

${ }^{16}$ Of course, this does not mean that $S$ never perceives $x$, but only that veridically perceiving an $x$ is not a mental property at all.

${ }^{17}$ Traditionally, this is how the causal theory of perception deals with perceptions: when $S$ perceives a worldly object $x, x$ is the cause of $S$ 's inner sensory experience, and this inner experience narrowly supervenes on $S$. Crucially, what distinguishes perceptions from hallucinations are the differences in their causal aetiology. A classical paper defending the causal theory of perception is Grice 1961. A more recent defence of it has been developed by Coates 2007.

${ }^{18}$ According to Johnston 2004, the common mental factor is a complex sensible profile that is instantiated by $x$ in veridical perception and uninstantiated in hallucination. For Dretske 1995, it consists of physical properties that are instantiated by $x$ in perception and uninstantiated in hallucination. 
the property of being $p$, the causal argument clearly shows that such a common mental factor makes it impossible for the presently existing worldly object $x$ itself directly to determine $S$ 's perceptual consciousness in perception.

That is, the real bite of the causal argument is twofold: i) it establishes the truth of CKA that is, in itself, already sufficient for showing that $\sim \mathrm{NR}$, and ii) it further makes plain that the narrowly supervening inner sensory experience $h$ exhausts the mental nature of perceptions and hallucinations. In the long run, both naïve and direct realism succumb to the causal argument and, therefore, have to be abandoned.

Keep in mind, however, that the causal argument is silent about whether sense-data, qualia, intentional objects, ways of sensing, or anything else, make up the metaphysical nature of the fundamental kind mentioned in CKA. Although quite distinct on the ontological footing, the causal argument emphasizes that these various perceptual accounts are right in introducing a mediating interface between mind and world. After all, the existence of such an interface in the first place provides the metaphysical ground making it intelligible to us why perceptual experiences, be they veridical or delusory, introspectively strike us as being occurrences of the same mental kind. ${ }^{19}$

\author{
Michael Sollberger \\ University of Lausanne \\ Department of Philosophy \\ CH-1015 Lausanne, Switzerland \\ michael.sollberger.2@unil.ch
}

\title{
References
}

Bickle, John \& Ralph Ellis. 2005. Phenomenology and Cortical Microstimulation. In Phenomenology and Philosophy of Mind, edited by D. W. Smith and A. L. Thomasson. Oxford: Clarendon Press, 140-163.

Burge, Tyler. 2005. Disjunctivism and Perceptual Psychology. Philosophical Topics 33: 1-78.

${ }^{19}$ The work on this paper has been supported by the Swiss National Science Foundation (SNSF), grant nr. 100011-117611. I am grateful to Michael Esfeld and Gianfranco Soldati for helpful suggestions and criticisms on earlier drafts of this paper. Thanks also to two anonymous referees for very helpful comments. 
Byrne, Alex \& Heather Logue. 2008. Either/Or. In Disjunctivism: Perception, Action, Knowledge, edited by A. Haddock and F. Macpherson. Oxford: Oxford University Press, 57-94.

Campbell, John. 2002. Reference and Consciousness. Oxford: Clarendon Press. Coates, Paul. 2007. The Metaphysics of Perception. New York: Routledge.

Dretske, Fred. 1995. Naturalizing the Mind. Cambridge, Mass.: The MIT Press.

Dunn, Jeffrey. 2008. The Obscure Act of Perception. Philosophical Studies 139: 367-393.

Einstein, Albert. 1948. Quanten-Mechanik und Wirklichkeit. Dialectica 2: 320-324.

Fish, William. 2008. Disjunctivism, Indistinguishability, and the Nature of Hallucination. In Disjunctivism: Perception, Action, Knowledge, edited by A. Haddock and F. Macpherson. Oxford: Oxford University Press, 144-167.

Fodor, Jerry A. 1987. Psychosemantics. Cambridge, Mass.: MIT Press.

Foster, John. 2000. The Nature of Perception. Oxford: Oxford University Press.

Gazzaniga, Michael S., Richard B. Ivry \& George R. Mangun. 2002. Cognitive Neuroscience: The Biology of the Mind. New York: W.W. Norton \& Company.

Grice, Herbert Paul. 1961. The Causal Theory of Perception. Proceedings of the Aristotelian Society, Supplementary Volume 35: 121-53.

Hawthorne, John \& Karson Kovakovich. 2006. Disjunctivism. Supplement to the Proceedings of The Aristotelian Society 80 (1): 145-183.

Hinton, J.M. 1967. Experiences. Philosophical Quarterly 17: 1-13.

Hinton, J.M. 1973. Experiences: An Inquiry Into Some Ambiguities. Oxford: Oxford University Press.

Howard, Don. 1985. Einstein on Locality and Separability. Studies in History and Philosophy of Science 16: 171-201.

Hume, David. 1740. A Treatise of Human Nature, edited by P. H. Nidditch. Oxford: Clarendon Press, 1978.

Johnston, Mark. 2004. The Obscure Object of Hallucination. Philosophical Studies 120: 113-183.

Langsam, Harold. 1997. The Theory of Appearing Defended. Philosophical Studies 87: 33-59.

Martin, M.G.F. 1997. The Reality of Appearances. In Thought and Ontology, edited by M. Sainsbury. Milan: FrancoAngeli, 81-106.

Martin, M.G.F. 2004. The Limits of Self-Awareness. Philosophical Studies 120: 37-89.

Martin, M.G.F. 2004b. Uncovering Appearances, unpublished manuscript: Michael Martin's UCL website: http://www.homepages.ucl.ac.uk/\%7Euctymfm/chapters.htm.

Martin, M.G.F. 2006. On Being Alienated. In Perceptual Experiences, edited by Tamar Szabo Gendler and J. Hawthorne. Oxford: Clarendon Press, $354-410$. 
McDowell, John. 1992. Putnam on Mind and Meaning. Philosophical Topics 20: 35-48.

McDowell, John. 1998. Criteria, Defeasibility, and Knowledge. In Meaning, Knowledge, and Reality, edited by J. McDowell. Cambridge, Mass.: Harvard University Press, 369-394.

Peacocke, Christopher. 1993. Externalist Explanation. Proceedings of the Aristotelian Society 43: 203-230.

Pitcher, George. 1971. A Theory of Perception. Princeton, NJ: Princeton University Press.

Robinson, Howard. 1985. The General Form of the Argument for Berkeleian Idealism. In Essays on Berkeley: A Tercentennial Celebration, edited by J. Foster and H. Robinson. Oxford: Clarendon Press, 163-186.

Robinson, Howard. 2001. Perception. London: Routledge.

Shoemaker, Sydney. 1980. Causality and properties. In Time and cause, edited by Peter van Inwagen. Dordrecht: Reidel, 109-135.

Siegel, Susanna. 2004. Indiscriminability and the Phenomenal. Philosophical Studies 120: 91-112.

Siegel, Susanna. 2008. The Epistemic Conception of Hallucination. In Disjunctivism: Perception, Action and Knowledge, edited by A. Haddock and F. Macpherson. Oxford: Oxford University Press, 205-224.

Smith, A.D. 2002. The Problem of Perception. Cambridge, Mass.: Harvard University Press.

Snowdon, Paul. 1981. Perception, Vision and Causation. Proceedings of the Aristotelian Society 81: 175-192. 